\title{
DE CIERTAS INSUFICIENCIAS \\ DE UN CIERTO LIBERALISMO (SEGUNDA RESPUESTA FEMINISTA A JORGE MALEM)*
}

1 final de mi artículo en el número 13 de $D o x a^{1}$ se celebraba la posibilidad de un diálogo entre personas que discuten desde posiciones ideológicas diferentes pero que comparten un sustrato común emancipatorio, lo cual obviamente se traduce en acuerdos pero también en desacuerdos. Con Malem comparto la aceptación del marco democrático-liberal en el que se desenvuelven nuestros sistemas políticos, pero no considero a ese marco, como supongo que él tampoco, ni constituido de una vez para siempre ni capaz por sí mismo de lograr la emancipación plena de los seres humanos que en él se integran. A efectos emancipatorios, lo más importante de los principios y de las doctrinas son los individuos y los colectivos que los formulan, los viven, los critican, los transforman y los profundizan en el sentido de una radicalización de la democracia y mayores cotas de participación. Con frecuencia, esto último es resultado de la confrontación política, económica, social y cultural de grupos que compiten entre sí para cambiar la condición y el lugar que la sociedad les ha asignado y con los que se encuentran disconformes. Si el mismo nacimiento de los estados liberales y su evolución es un producto sociohistórico, en el marco de las libertades básicas formales en que nos movemos tienen cabida desde la Nueva Derecha norteamericana de los años ochenta o los regímenes socialdemócratas de los países nórdicos europeos hasta la experiencia democrático-revolucionaria fallida (en parte por las maniobras de los «liberales» del Norte) de

* Los artículos de Malem a que aquí se alude son los siguientes: «Pornografía y feminismo radical» (Doxa . $^{\circ}$ 12) y «Feminismo radical, pornografía y liberalismo» (Doxa n. $^{\circ} 13$ ). Para saber a cuál de ellos nos estamos refiriendo, a la página citada le añadiremos las letras $\mathrm{A}$ y $\mathrm{B}$ respectivamente.

${ }^{1}$ Raquel Osborne, «Liberalismo y feminismo: ¿Un dilema para las mujeres? Respuesta a Jorge Malem», Doxa, 13, 1993. 
un Salvador Allende en el Chile de comienzos de los setenta. Es decir, los principios se entenderán y funcionarán de manera muy diferente según la interpretación que les otorguen los sectores en liza.

La existencia de los movimientos sociales, posible en este contexto de libertades democráticas, resalta algunas de las insuficiencias de los regímenes democrático-liberales. Los principios de igualdad y libertad no son nada si no se les dota de contenido -cualquier dictador habla sin empacho de libertad y la igualdad adquiere un significado extravagante en el contexto de una política económica ultraliberal-. El ecologismo ha visto que el sistema económico nacido de la revolución industrial destruye el medio ambiente; desde el pacifismo se ha llegado a la conclusión de que se debe luchar por la paz si se pretende que el mundo sobreviva a causa de la potencia autodestructiva generada por el ser humano; los movimientos homosexuales han luchado por legitimar sus opciones sexuales cuestionando los modelos heterosexuales dominantes; las mujeres se han organizado en el movimiento feminista para lograr, no sólo unos derechos formales y su aplicación real, sino la transformación del sistema patriarcal que jerarquiza y segrega a los géneros, creando desigualdad social donde sólo hay diferencia sexual.

El feminismo ha nacido, pues, de la contradicción entre la proclamación formal de unos principios y su negación en la práctica para las mujeres. El sufragio no se ha constituido como un derecho universal hasta el siglo veinte, tras largas e intensas luchas por parte de las feministas de la primera ola, las sufragistas. A partir de los años sesenta, por motivos que no hace al caso mencionar aquí, surge de nuevo en primer plano la contradicción mencionada y aparece el movimiento feminista contemporáneo, Como la discusión entre Malem y yo se ciñe fundamentalmente a polémicas y hechos acaecidos en los EE.UU., referiré a aquel país los datos que a continuación expongo. No trataré en esta segunda respuesta todos los aspectos que podrían ser susceptibles de comentarios por mi parte porque entonces la discusión sería interminable; creo que las/os lectoras/es me lo agradecerán. Algunas cuestiones de fondo servirán para el propósito de la discusión.

Malem se queja de que yo rechazo su utilización de la expresión «feminismo radical» pero que no menciono su caracterización de lo que denota ese término. Tiene razón, pero mi omisión obedece a que me ha resultado imposible colegir de su primer texto qué entendía por feminismo radical, como no fuera el movimiento antipornografía. Lo más aproximado a una caracterización por su parte han sido retazos como los siguientes: «El feminismo radical es, sin duda, un declarado enemigo de la pornografía» (pág. 177A); «... las feministas radicales -autoproclamadas de izquierdas-... (págs. 177-78A)»; «En 
realidad, bajo el epígrafe "feminismo radical" se hace referencia a un conjunto de grupos que si bien participan de un cierto aire de familia mantienen algunas diferencias entre sí (nota 4 pág. 180A)».

Por mi parte, pienso que si se reprocha con tanto ardor al interlocutor que no ha acotado con precisión el ámbito del debate, conviene ser consecuente con las propias exigencias y tratar de situar histórica e ideológicamente el movimiento que decimos que vamos a criticar. Dado que Malem no se siente muy inclinado a hacerlo, suministraré brevemente a las/os lectoras/es algunas pinceladas sobre este feminismo a fin de que, de una vez por todas, sepan de qué estamos hablando. Dado que considero imprescindible contextualizar algo más la polémica, me serviré de la nomenclatura que me parece más precisa para la designación de los diversos grupos que deben ser mencionados, abandonando mi anterior dicotomía «feminismo pro censura/feminismo anticensura», quizás en exceso simplificadora y en definitiva susceptible de mayores matizaciones si se me permite ampliar el cuadro de mi análisis.

Como muestra del «estado de indigencia teórica que padece el feminismo radical» (pág. 186A), habría que mencionar que dentro de lo que se denominó «el movimiento de liberación de las mujeres», surgido en los EE.UU. a mediados de los años sesenta, se distinguieron nítidamente tres corrientes feministas: feminismo liberal, feminismo radical y feminismo socialista. El feminismo radical se diferenciaba del feminismo socialista porque no consideraba al capitalismo como la principal fuente de opresión de las mujeres y no confiaba en el advenimiento del socialismo para luchar por su emancipación, discrepando a su vez de la solución propuesta por el feminismo liberal, consistente simplemente en la integración de las mujeres en la esfera de lo público. Para las radicales, la contradicción principal radicaba en el género antes que en la clase social, y las relaciones entre las mujeres y los hombres necesitaban ser redefinidas en términos políticos. Desvelaron que la igualdad formal era insuficiente en una sociedad marcada por el racismo y la estratificación social y resaltaron que la desigualdad entre las mujeres y los hombres en la esfera de lo público se hallaba relacionada con la subordinación femenina en la familia².

Según Ellen Willis, «las feministas radicales acuñaron los términos de "sexismo" y "política sexual"... para expresar la idea de que la sexualidad, la vida familiar y las relaciones entre hombres y mujeres no eran simplemente el resultado de una elección individual, o incluso de la costumbre, sino que incluían el ejercicio del poder per-

${ }^{2}$ Alice Echols, Daring to Be Bad. (Radical Feminism in America 1967-1975), Minneapolis: University of Minnesota Press, 1989, pág. 3. 
sonal e institucional y suscitaban cuestiones vitales de política pública» ${ }^{3}$. El sexismo no era la expresión natural de las diferencias sexuales «sino un sistema social incrustado en el derecho, la tradición, la economía, la educación, la religión organizada, la ciencia, el lenguaje, los medios de comunicación, la moralidad sexual, la crianza de los niños, la división doméstica del trabajo y la interacción social cotidiana, cuya intención y efecto era dar poder a los hombres sobre las mujeres» ${ }^{4}$.

Hacia 1975 el movimiento feminista radical desapareció como tal. Su sentido político originario fue oscurecido por lo que Alice Echols denominó con fortuna el feminismo cultural, cuya visión representó un alejamiento tan fundamental respecto de la óptica primera del feminismo radical que pareció aconsejable diferenciar a uno de otro. Ella usa el término «feminismo radical» para describir los primeros pasos del movimiento 5 , mientras que a esta versión más reciente de aquel feminismo prefiere caracterizarla como «feminismo cultural». Según Echols ${ }^{6}$, el feminismo radical fue un movimiento dirigido a la eliminación del sistema de dominación patriarcal, mientras que el feminismo cultural identifica la liberación de la mujer con el desarrollo y preservación de la cultura femenina, a base de ensalzar y primar los «valores femeninos» y exorcizar y devaluar los «valores masculinos». En otras palabras, el feminismo radical partía de un enfoque social constructivista que aspiraba a convertir al género en una categoría irrelevante mientras que las feministas culturales se decantaban por un esencialismo que se complacía en la celebración de «lo femenino». Para decirlo con una terminología más habitual en España, las primeras movilizarían a las mujeres sobre la base de su semejanza con los varones y las segundas lo harían insistiendo en el principio de la diferencia femenina.

Como sostengo en mi libro La construcción sexual de la realidad ${ }^{7}$, las elaboraciones del feminismo cultural, que toman cuerpo en unos años de creciente reacción conservadora en los USA, contribuyeron en buena medida al clima de ideas que a finales de los setenta cristalizaron, entre otras cosas, en el feminismo antipornografia, el feminismo que se organizó en grupos dedicados exclusivamente a la lucha contra el fenómeno de la pornografía.

${ }^{3}$ Ellen Willis, «Foreword», en Alice Echols, ibíd., pág. VII.

${ }^{4}$ Ibíd., págs. VII y VIII.

${ }^{5}$ Alice Echols, «The New Feminism of Yin and Yang», en Ann Snitow, Christine Stansell y Sharon Thompson, eds., Powers of Desire. The Politics of Sexuality, Nueva York: Monthly Review Press, 1983, págs. 439-59.

${ }^{6}$ Alice Echols, Daring.... op. cit., pág. 6.

${ }^{7}$ Raquel Osborne, La construcción sexual de la realidad, Madrid: Cátedra, 1993. 
Ya en 1969 el feminismo radical concebía a la pornografía como problemática para las mujeres, pero las diferencias entre sus planteamientos originarios y los del (feminismo cultural convertido en) movimiento antipornografía en la segunda década de los setenta son notables. En los comienzos, en efecto, se combatía la pornografía pero conjuntamente con otras representaciones de las mujeres en los medios de comunicación: la pornografía no era central para el análisis de la dominación masculina ni constituía el meollo de la opresión de las mujeres. En el análisis posterior, por contra, dicha opresión se hizo descansar en una visión altamente dicotomizada de la sexualidad masculina -fundamentalmente un instrumento de dominación sobre las mujeres- y la femenina -proclive a la reciprocidad y a la intimidad en sus relaciones sexuales-, lo cual delataba una caracterización de las categorías de lo femenino y lo masculino como cosificadas e inmutables que, según esta óptica, se vería no sólo re-creada por sino fundamentada en la pornografía. Por lo demás, desde el movimiento feminista radical se favorecían los boicots y las protestas en contra de las representaciones sexistas en los media en vez del uso de la maquinaria legal del Estado para limitar o eliminar la pornografía.

En resumen, contamos con los siguientes datos: el movimiento feminista radical conserva una notable fuerza dentro del panorama del feminismo norteamericano hasta la primera mitad de los años setenta, en que los grupos que lo integraban desaparecen y muchas de sus ideas fueron notablemente transformadas por un conjunto de autoras feministas, pasándose a planteamientos esencialistas acerca de la concepción de los géneros. Este fenómeno ha sido estudiado en profundidad y el nuevo conjunto de ideas recibió la denominación de feminismo cultural. Dichas ideas cristalizan, en su forma más categórica, en el movimiento feminista en contra de la pornografía, conjunto de grupos que, con el ideario del feminismo cultural, se constituye con aquel nombre para luchar contra lo que en su creencia constituye el principal motivo productor-inductor de la opresión de la mujer: la pornografía.

Algunas de las mujeres que lo abanderan provienen de las filas del feminismo radical pero han desarrollado las ideas del feminismo cultural. Otras simplemente han adoptado este último ideario y lo han aplicado al tema de la sexualidad y la pornografía.

Otra corriente feminista, heterodenominada pro-sexo, o también anti-censura, incluye mujeres que asimismo provienen del movimiento feminista radical, y que han permanecido fieles a un ideario no esencialista y de no intervencionismo legislativo. Se nutre igualmente de mujeres procedentes de los otros dos feminismos identificados más arriba -liberal y socialista- o que simplemente han reco- 
gido -y elaborado- las ideas del movimiento feminista radical, dado que uno de los rasgos de este último es el de su desaparición física pero no la evaporación de su legado, que ya forma parte -con todas las elaboraciones y críticas necesarias, repito- del acervo feminista general. A la larga, el principal logro de estas ideas ha sido «la destrucción de los parámetros tradicionales acerca de las relaciones entre mujeres y hombres ${ }^{8}$. Esta corriente ha liderado en la sociedad norteamericana la confrontación de ideas y acciones con el feminismo antipornografía, organizándose incluso durante un tiempo bajo las siglas de FACT -Organización feminista contra la censura- para, en alianza con la ACLU (Sindicato a favor de las libertades civiles) y gremios de libreros y editores entre otros, contrarrestar las iniciativas pro-censura de Catharine MacKinnon y Andrea Dworkin y de sus aliados conservadores.

Como, a pesar de lo que opina Malem, hay quienes piensan que la herencia teórica del feminismo radical es considerablemente rica, es posible que algunas de las feministas antipornografía se autodenominen feministas radicales, tratando así de capitalizar en provecho propio aquel caudal. Pero para quien dice estudiar con profundidad un fenómeno y proclama la necesaria acotación del objeto de estudio, todas las anteriores especificaciones han de tenerse muy en cuenta so pena de correr el riesgo de falsear la realidad. Si desde el propio movimiento feminista se ha estudiado en profundidad los avatares del movimiento feminista radical -el libro de Alice Echols, ya citado, fue su tesis doctoral-, ¿cómo pasar por alto el fruto de sus análisis? Según éstos, las heterodenominadas feministas prosexo o anticensura serían las más fieles herederas del ideario original del feminismo radical por las razones que acabo de exponer en los párrafos anteriores. Y como resulta ya meridianamente claro a estas alturas, nadie antes que ellas, ni tan extensamente como ellas, ni desde dentro como ellas, han criticado al feminismo antipornografía, así en sus presupuestos ideológicos como en su práctica política de intenciones represoras. Es decir, la crítica de la inequívoca proclividad hacia posturas conservadoras de un cierto sector del movimiento socio-político de inspiración feminista ha sido liderada por sus propias compañeras de movimiento.

Malem, pues, se halla legitimado para comentar desfavorablemente la actuación de un subsector del movimiento feminista como es el feminismo específicamente organizado para luchar contra la pornografía, pero haría bien en delimitar el blanco de sus críticas en lugar de atrapar al vuelo la primera etiqueta que aparece por ahí y disparar como quien descubre la pólvora contra objetivos que ya lo

${ }^{8}$ Willis, op. cit., pág. X. 
eran de las auténticas herederas del feminismo radical, que ni siquiera necesitan seguirse etiquetando de tales a estas alturas, puesto que el movimiento en sí hace tiempo que ha desaparecido. En cuanto a la crítica propiamente feminista de aquellos objetivos, no ha procedido solamente en el plano teórico, de las ideas, sino en el de la militancia, en el terreno de la praxis, organizándose para combatir iniciativas de corte proteccionista-represivo. Puesto que acusa de indigencia teórica al feminismo radical -y lo hace, según vemos, sin saber muy bien de qué está hablando-, Malem debería tomar nota de todo lo antedicho por dos razones cuando menos, además de la de zafarse del calificativo de indigente a esos efectos: la primera porque las principales ideas manejadas por el así denominado feminismo prosexo provienen del ideario del movimiento feminista radical -con la evolución lógica que marca el paso del tiempo- aplicado al objeto que nos ocupa, y la segunda, concatenada con la anterior, porque el grueso de la argumentación de nuestro filósofo del derecho tanto en contra de la censura como en contra de los postulados ideológico morales del feminismo antipornografía, sean cuales fueren las fuentes que maneje, ya había sido desarrollado en los escritos de las feministas prosexo. Cronología obliga.

En mi anterior trabajo se formulaba la hipótesis de que la postura sostenida por Malem podía degenerar, «queremos pensar que inconscientemente, en antifeminista» ${ }^{9}$. Pues bien, creo que en el intervalo entre el primero y el segundo artículo de Malem se ha producido un acelerado proceso de «degeneración» y su posición se ha tornado meridianamente diáfana, cosa por otra parte muy de agradecer. Dada su confusión acerca de lo que entiende como feminismo radical, no le puedo reprochar que se manifieste «en contra de los presupuestos teóricos, de ciertas actitudes políticas y de la posición moral» (pág. 314B) de lo por mí entendido como feminismo antipornografía. Pero a continuación amplía su comentario a los «diversos feminismos»: «Se puede incluso estar en contra de la mayor parte de los aspectos teóricos de los diversos feminismos, y sin embargo, estar a favor de los derechos que como seres humanos corresponden a las mujeres. Feminismo y mujeres no son términos sinónimos, ni coextensivos. Ni las feministas tienen el patrimonio de la defensa de los derechos de las mujeres» (ibíd.). No, claro, ahora va a resultar que dicho patrimonio les está encomendado a varones como Malem que sí saben cuáles son los verdaderos derechos humanos que nos corresponden y proceden a contárnoslo a las feministas que, a causa de nuestra indigencia teórica, no acabamos de entrever en qué consisten nuestros genuinos intereses. Lo mismo ocurre, por supuesto,

${ }^{9}$ Osborne, «Liberalismo...», op. cit., pág. 289. 
con otros sectores oprimidos: los blancos le tienen que decir a los negros cuáles son sus verdaderos derechos, los heterosexuales a los homosexuales, y así sucesivamente.

«Un ataque a los presupuestos teóricos del feminismo (a secas, de nuevo) -en sus diversas vertientes- en modo alguno implica un ataque a las mujeres» (ibid.). Si el sistema de sexo-género, o el sistema patriarcal, establece unas relaciones de dominación y subordinación entre hombres y mujeres, estas últimas habrán de organizarse para modificarlas, y eso es lo que el feminismo ha hecho: crear un movimiento para que las mujeres se dieran cuenta colectivamente de su situación y pensaran en salir de ella. El sistema, como ya hemos señalado, se incardina en todas las instituciones: «en el derecho, la tradición, la economía, la educación, la religión organizada, la ciencia, el lenguaje, los medios de comunicación, la moralidad sexual, la crianza de los niños, la división doméstica del trabajo y la interacción social cotidiana», cuya intención y efecto es «dar poder a los hombres sobre las mujeres» ${ }^{10}$. Por ello hay que desasirse del yugo masculino y, por la misma razón, los varones no son los más indicados para decirnos lo que debemos hacer. Los varones tendrían que compartir la lucha feminista por la emancipación de las mujeres para recibir otra consideración, la de aliados, que pueden ser aliados críticos pero no «atacantes» -pág. 314B- que ignoran los matices en liza -omisión de mencionar al feminismo pro-sexo o anticensura- y que cuando «atacan» piensan que su interlocutora u oponente les ve a ellos como enemigos -pág. 301B- (¿Lapsus freudiano? Debe ser que Malem está en guerra con las feministas pero yo no me había enterado). Por eso sí merece la pena entrar en una discusión por «una mera cuestión de nombres», porque lo que se desvela es que las divergencias son de fondo y conviene ponerlas en primer plano para no dejarlas difuminarse tras cortinas de humo.

Siguiendo en esta tónica de resaltar las divergencias de fondo, los «ataques» de Malem me han hecho cavilar durante todo este tiempo en las razones que le moverían a ello. Yo no pretendo «la eliminación del Estado democrático» ni comparto la mayoría de las posiciones de alguien como Catharine MacKinnon en su caracterización de la pornografía ni en su forma de limitar los posibles efectos negativos de esta última. Mi divergencia con las posturas de MacKinnon no es meramente metodológica o siquiera teórica, sino que me produce particular desazón que alguien que se proclama participante de un movimiento que pretende la emancipación de las mujeres sostenga ideas y promulgue políticas en mi opinión contraproducentes a esa emancipación. ¿Por qué no trata Malem de enfatizar lo que nos

${ }^{10}$ Willis, op. cit., págs. IX y X. 
une y no de subrayar lo que nos separa, o por qué trata repetidas veces de meterme en el mismo -o parecido- saco que a MacKinnon? Creo que la única respuesta plausible es la de porque le interesa resaltar lo que nos une a ambas y que a él le distancia de nosotras: nuestro feminismo y su antifeminismo.

Pero Malem comete en este sentido un craso error con mi análisis de la situación y es que yo no estoy pretendiendo utilizar el derecho del Estado de Derecho para combatir la mayoritaria pornografía al uso producida y consumida por y para varones en esta sociedad. MacKinnon sí, y por eso incurre en esa contradicción que yo ya resaltaba -estar en contra del Estado liberal-democrático pero intentar utilizar sus herramientas legales para sus finesy que la hace tan vulnerable a críticas filosófico-legalistas. Desde la posición que yo mantengo no se pretende establecer ninguna nueva definición legal para limitar o erradicar lo que quiera que entonces se considerara pornografía. Se estaría en desacuerdo con la consideración dominante, en cuanto que reflejada en las leyes, de que la explicitación sexual de algunos materiales los convierte en «obscenos» y por ello son susceptibles de control legal. Pero esta crítica no va dirigida hoy por hoy a un cambio del modelo legal, como intenta MacKinnon, sino que se manifiesta como una crítica de la cultura, de la mentalidad, de las ideas que conciben de esta manera la obscenidad. A quienes observan estos criterios no les importa la manipulación y exhibición de los cuerpos femeninos en un contexto que los pone al servicio de un modelo de sexualidad hecho para el disfrute de los varones y que apela fundamentalmente a sus fantasías. Al moverse en este terreno, no hay por qué ceñirse al estricto marco del daño al que nos veríamos obligadas por el Estado de Derecho si se exige una intervención legal restrictiva. Y se pueden destacar los daños, los perjuicios, las consecuencias negativas para las mujeres que parezcan más oportunas porque se está tratando de influir en el terreno de las ideas, de las mentes. Se intentará persuadir y convencer con todos los argumentos posibles, pero no habrá que ajustar las afirmaciones a ningún marco legal. Por eso resulta legítimo tachar algunos materiales de sexistas, o degradantes, y definir sexismo y degradación como mejor parezca para cumplir los fines deseados: el intento de ser comprendidos, y de ahí que las explicaciones se afanen en lograrlo. En consecuencia, los intentos de Malem de asimilar esta postura a la de MacKinnon o criticar la noción de daño en los términos expuestos se convierte en una impostura porque nos estamos situando en otro plano diferente del legal, cosa que no parece haber entendido Malem, quizás por su formación jurídica, pero conviene resaltar a fin de que se sepa que hay otras cosas en esta sociedad además de las leyes o el derecho. 
En este sentido no sé a qué viene la alusión al principio del daño en conexión con el «coto vedado» y la lección acerca de que no son necesarios «nuevos y vagos principios... ni un nuevo Estado diferente del democrático y social basado en la igualdad y la libertad...» (pág. 309B). Ésta es la amalgama en la que Malem quiere aunar a muy diversas feministas para así descalificar al movimiento en su conjunto. Es como cuando se afirma que «todos los negros son iguales» o «a los judíos se les reconoce por su nariz ganchuda». ¿Es que todas las feministas atacamos el derecho a la libertad de expresión en la línea expresada por MacKinnon? Ciertamente no, e incluso muchas se han manifestado activamente en contra de semejante postura. Con todo, el binomio igualdad-libertad no siempre se halla en perfecta consonancia en cuanto a la situación de las mujeres se refiere, y, en el caso que nos ocupa, los contenidos de la libertad no siempre se distinguen por su inclinación a la igualdad entre los sexos, puesto que el modelo sexual al uso por el que se guía la pornografía no es precisamente un modelo igualitario. La pornografía mayoritaria presenta los cuerpos de las mujeres siempre disponibles y expuestos a la mirada y fantasía masculinas, y esto responde a, y redunda en, un modelo de sexualidad que prima la iniciativa y la cantidad -para los varones- mientras que a las mujeres se nos exige esta perpetua disponibilidad al tiempo que se nos castiga con el apelativo de putas si acaso nos manifestamos como iniciadoras o promiscuas.

Como ya señalaba en mi artículo, esta mentalidad de servicio es común a todos los discursos científicos y culturales de los últimos cien años en tomo a la sexualidad, con la peculiaridad de que en el caso de la pornografía este servicio se presenta como exclusivamente sexual. Es decir, la crítica a la pornografía se insertaría, desde esta perspectiva, en el marco de una crítica feminista de la cultura, y la pornografía sería un elemento más de esa cultura, no, como cree MacKinnon, el centro principal, desde donde irradia nuestra subordinación. Por lo tanto es perfectamente válida la crítica a aquello que desde esta óptica se evalúa como el sexismo de muchas de las representaciones femeninas en la pornografía al uso, sin que produzca entusiasmo la forma en que se utiliza la libertad de expresión para comercializar el sexo de esa manera -como un medio para la satisfacción masculina y no como una vía para crear sujetos autónomos-, pero se sobreentiende que la libertad de expresión es muy cara a las mujeres y no se desea prescindir de ella. Desde el feminismo, pues, se cuestiona ese supuesto e idílico estado de igualdad y libertad para las mujeres en relación con los hombres en esta sociedad, pero mientras hay quienes, como MacKinnon, pretenden disminuir las competencias del coto vedado por entender que sólo sirve para apuntalar la 
libertad de algunos en detrimento de la igualdad de todos, otras prefieren «ensanchar el recinto de aquel coto» a fin de poder modificar y dar cabida a todas las ideas que desde el feminismo disienten de las predominantes, para decirlo al modo de Javier Muguerza ${ }^{11}$.

En cuanto al eslogan de que lo personal es político, ya he comentado en parte su sentido al comienzo de este trabajo, pero convendría quizás detallar algo más sus implicaciones para evitar malentendidos como los que parecen afectar a Malem. Con estos conceptos se trata de establecer los términos de una relación, señalar que existe conexión entre los dominios de lo público y lo privado en la vida social y destacar que no son compartimentos estancos sin nada que ver el uno con el otro. De esta forma se puede evitar la caída en una postura individualista a ultranza que sólo tiene en cuenta la sexualidad como si ésta se diera en un vacío social, postura tras la que se ocultan las diferencias de poder. En el otro extremo se situaría una posición totalitaria de la sexualidad que la ve como determinada absolutamente por el todo social, sin margen posible para la autonomía o la decisión individual ${ }^{12}$.

En el caso de la pornografía, y si nos referimos por ejemplo a las mujeres que trabajan en ella, una visión totalitaria a lo MacKinnon viene a sostener que en una sociedad como la actual, en la que se objetualiza sexualmente a la mujer, ninguna mujer puede «escoger» voluntariamente «ser» un objeto sexual, y que por tanto la distinción entre trabajo por consentimiento o a la fuerza deviene irrelevante. La mejor respuesta a esto es la que dan las propias implicadas en estas tareas cuando proclaman su decisión individual de trabajar en la industria del sexo como la mejor opción a su alcance, afirmando, eso sí, su «derecho a ser sujeto de derechos» ${ }^{13}$, que les son sustraídos por la estigmatización a que se ven sometidas sobre todo las mujeres que trabajan en aquella industria. Pero la posición feminista no deja de reconocer, por citar algunos aspectos de la cuestión, que en dicha industria las mujeres suelen carecer de poder alguno tanto en la producción como en la distribución del producto, no teniendo, en consecuencia, poder para controlar las relaciones laborales ni el producto final; que las mujeres en esta sociedad cuentan con muchas menos y peores opciones laborales que los varones, lo que comporta que su

${ }^{11}$ Javier Muguerza, «Primado de la autonomía: ¿Quiénes trazan las lindes del "coto vedado"?», en Jorge Malem, comp., Homenaje a Ernesto Garzón Valdés (en prensa), pág. 20.

${ }^{12}$ Mariana Valverde, «Beyond Gender Dangers and Private Pleasures: Theory and Ethics in the Sex Debates», Feminist Studies, vol. 15, n. ${ }^{\circ}$ 2, verano de 1989, págs. 237-254, esp. pág. 250.

${ }^{13}$ Muguerza, op. cit., pág. 20. 
margen de elección se vea mucho más reducido que el de éstos; y que una mujer puede verse estigmatizada por efectuar este trabajo, mucho más de lo que los hombres implicados en el mismo puedan serlo a causa de la manera en que la diferente y jerarquizada consideración social entre hombres y mujeres influye cuando lo hacen, como se comenta en mi ya citado libro La construcción sexual de la realidad ${ }^{14}$. Entender por tanto que ambos aspectos de lo público y lo privado se hallan implicados mutuamente no hace sino enriquecer el análisis, favoreciendo matizaciones que permitan respetar la libertad personal de los individuos pero que al mismo tiempo pongan de manifiesto que entre mujeres y varones no reinan sin más la libertad y la igualdad.

MacKinnon, a su vez, rechaza que cualquier noción de privacidad sea positiva para las mujeres. Malem, al efectuar reiteradamente la maniobra de alinearme con sus posiciones para tratar de descalificarme, no matiza nuestras diferencias cuando insiste en que desde el feminismo se critica la noción de privacidad esgrimida por el Tribunal Supremo de los Estados Unidos para garantizar y/o restringir, según los momentos, el acceso al aborto en aquel entorno. Pero no tiene en cuenta que MacKinnon aprovecha esta instancia para atacar frontalmente al liberalismo y negarle toda validez para las mujeres, mientras que desde otras posturas se analizan las insuficiencias del liberalismo, pero no se cuestiona el marco liberal democrático en el que nos desenvolvemos. Es una diferencia clave dentro del feminismo -y no sólo dentro de éste- y que sin embargo Malem pasa por alto en este caso como en tantos otros.

Las sentencias Roe v. Wade (1973) y Harris v. McRae (1981) se mueven en direcciones opuestas en cuanto a la expansión -la primera- o constricción -la segunda- del «coto vedado», entendido en esta ocasión como el derecho al aborto. Yo no he pretendido en ningún momento establecer una conexión causal entre una u otra sentencia, como Malem me atribuye, pero sí hacer constar que, según quien interprete ciertos principios o nociones, los mismos pueden, en determinados contextos y encapsulados en ciertas estructuras, ser utilizados a favor o en contra de las mujeres. Desde un punto de vista feminista, Roe v. Wade, a pesar de ser ampliamente celebrada por las mujeres como una victoria, no se correspondía exactamente con sus planteamientos. Me servirá de guía la argumentación de Rosalind Petchevsky en su exhaustivo análisis de las relaciones entre el Estado, la sexualidad y la libertad reproductiva ${ }^{15}$.

\footnotetext{
${ }^{14}$ Osborne, La construcción.... op. cit., págs. 199-200.

${ }^{15}$ Rosalind Pollack Petchesky, Abortion and Woman's Choice. (The State, Sexuality, and Reproduction), Boston: Northeastern University Press, 1985.
} 
En ningún momento, ni siquiera en el caso de Roe v. Wade, la despenalización del derecho al aborto se hizo en el marco de las nociones feministas de un derecho y una necesidad social para todas las mujeres. En su lugar, fue conceptualizado como una prerrogativa médica o como un abstracto derecho a la privacidad que, en la práctica, ha excluido a las demasiado pobres o demasiado jóvenes para poder ejercer dicho derecho sin la ayuda pública. No era el aborto a petición de las mujeres (abortion on demand) lo que ahí se reconocía, ni tan siquiera en el primer trimestre del embarazo, sino más bien que «la decisión del aborto, en todos sus aspectos, es inherente y primordialmente una decisión médica, y la responsabilidad básica sobre ello debe recaer en el médico». El mismo concepto de aborto terapéutico implica que los abortos fuera de ese estricto marco médico no son necesarios, oscureciendo la idea de que los motivos familiares, económicos, sociales o sexuales, que a su vez se hallan relacionados con aspectos médicos, constituyen una justificación suficiente para abortar. Estos comentarios no desconocen que el marco normativo establecido en Roe v. Wade, que ponía el criterio de la salud de las mujeres, en su más amplio sentido, por encima de un abstracto moralismo o de los derechos del feto, constituía seguramente la vía más práctica para convertir en viable la concreción del derecho al aborto en el contexto del sistema sanitario tal y como se halla estructurado. Pero la sentencia abría igualmente las puertas a la intervención estatal (en sentido restrictivo, como sucedería posteriormente) cuando afirmaba que «la mujer embarazada no puede ser aislada en su privacidad. Porta en sí misma un embrión y, posteriormente, un feto... Su situación es, por tanto, inherentemente diferente de la intimidad marital, o de la posesión de material obsceno en el dormitorio, o del matrimonio, o de la procreación...» ${ }^{16}$.

Al definir el aborto como un abstracto derecho a la privacidad individual no se tenían en cuenta las situaciones reales en que muchas mujeres no podían alcanzar dicho derecho, aunque las leyes así lo reconocieran formalmente. La cláusula de conciencia es un ejemplo por todas/os conocido, que comporta (tanto allá como aquí) grandes disparidades en cuanto a la accesibilidad al aborto en distintas partes del país. La invocación del derecho a la privacidad como la máxima justificación del derecho al aborto puede resultar equívoca puesto que se presta a interpretaciones que favorecen las demandas profesionales y empresariales de los médicos -Roe v. Wade confirmaba legalmente el modelo de confidencialidad médico-paciente ya existente en la práctica clínica de las mujeres de clase media, pero no afirmaba nada acerca del derecho de las mujeres a recibir este

${ }^{16}$ Roe versus Wade, pág. 159, apud., pág. 292. 
tipo de atención médica-. Del mismo modo, la apelación a la privacidad resalta el carácter personal e individual del aborto dejando de lado la idea de que el derecho al aborto corresponde a todas las mujeres en tanto colectivo, y que no entenderlo así supone un perjuicio para dicho colectivo. Por añadidura, la negación de una base social que justifique el aborto como una necesidad o un derecho de las mujeres, el entenderlo como una «elección privada» antes que como un prerrequisito para una vida decente, contribuye a perpetuar las divisiones de clase entre las mujeres. En una sociedad dividida por clases sociales, dejar a las personas sujetas a sus propios recursos económicos implica privar a muchas del acceso a ciertos derechos.

Cuando hacia 1977 el péndulo de la política se había inclinado hacia la derecha y el Congreso se hallaba discutiendo las provisiones de la Enmienda Flyde para restringir la financiación pública de los abortos, el Tribunal Supremo respondió en el mismo sentido y una serie de sentencias culminaron en Harris v. McRae. Por medio de esta última se consagraban las intenciones de la citada enmienda de no proporcionar los medios materiales necesarios para ejercitar su libertad de elección a aquellas mujeres que lo necesitaran; el Estado no se hacía responsable de las desigualdades económicas puesto que se afirmaba que la justicia social no corresponde a los tribunales, sino a los intereses que compiten a la hora de determinar la política social. En consecuencia, el derecho al aborto no quedaba determinado por la decisión de la mujer sino por su acceso a los recursos privados. Si el aborto hubiera sido despenalizado como un derecho derivado de las necesidades de las mujeres y no como un derecho a la privacidad susceptible de variopintas interpretaciones, el Tribunal Supremo habría tenido que crear esta doctrina ex novo y no, como sucedió, aprovechar los pasos previamente dados para dejar lo más vacío posible de contenido un derecho de las mujeres.

Éste, y no otro, sería el sentido de algunas críticas feministas a los criterios que orientaron inicialmente la despenalización del aborto, que según estos argumentos, abría las puertas para que no se tuviera suficientemente en cuenta las necesidades reales de las mujeres. MacKinnon, cuando se da cuenta de esas insuficiencias, reniega del liberalismo, mientras que desde posiciones a las que me siento más próxima se apunta más bien a un libertarismo en el sentido muguerziano del término, es decir, de radicalización de la democracia, según vendría a desprenderse de mi anterior respuesta.

\section{DOXA 17-18 (1995)}

